\title{
Design and Characterization of a 2-DOF MEMS Ultrasonic Energy Harvester With Triangular Electrostatic Electrodes
}

\author{
Anthony G. Fowler, Student Member, IEEE, S. O. Reza Moheimani, Fellow, IEEE, \\ and Sam Behrens, Member, IEEE
}

\begin{abstract}
This letter presents a microelectromechanical systems (MEMS) based energy harvester designed for applications such as the powering of implanted biomedical devices. The harvester is mechanically excited by an external source of ultrasonic waves with triangular electrostatic electrodes being used to produce electrical power from the vibrations of the system's proof mass. Comparisons are made with a previous MEMS harvester that uses conventional comb-finger electrostatic electrodes to demonstrate that the triangular electrodes result in a more effective conversion process. Experimental characterization shows that the device produces an average power output of $27.6 \mathrm{nW}$, which corresponds to a $29 \%$ increase in power output over the conventional comb-finger device.
\end{abstract}

Index Terms-Electrostatic transducer, energy harvesting, energy scavenging, microelectromechanical systems (MEMS).

\section{INTRODUCTION}

$\mathbf{E}$ NERGY harvesting continues to be an area of particular relevance for many applications involving batterypowered electronic devices. Portable systems, such as wireless sensor nodes, laptops, and mobile phones, have a limited run time due to their reliance on a battery with a finite capacity [1], [2], and thus the ability to convert other forms of energy, including heat and vibrations into usable electrical power is an attractive prospect. Microelectromechanical systems (MEMS)based devices have commonly been used for energy harvesting applications [3] with their relatively straightforward fabrication and ease of integration with other electronic systems making them ideal for this purpose.

The powering of implanted biomedical devices is a particularly useful application for microscale energy harvesters. Implanted devices, such as embedded diagnostic sensors and pacemakers, continue to rely on batteries as their primary power source, and thus there is a continuing interest in developing methods of reliably and safely transmitting power

Manuscript received June 21, 2013; revised August 23, 2013; accepted September 15, 2013. Date of publication October 9, 2013; date of current version October 21, 2013. This work was supported in part by the Australian Research Council and in part by the Commonwealth Scientific and Industrial Research Organisation. The review of this letter was arranged by Editor S. J. Koester.

A. G. Fowler and S. O. R. Moheimani are with the School of Electrical Engineering and Computer Science, University of Newcastle, Callaghan 2308, Australia (e-mail: reza.moheimani@newcastle.edu.au).

S. Behrens is with CSIRO Energy Technology, Newcastle 2300, Australia.

Color versions of one or more of the figures in this letter are available online at http://ieeexplore.ieee.org.

Digital Object Identifier 10.1109/LED.2013.2282815

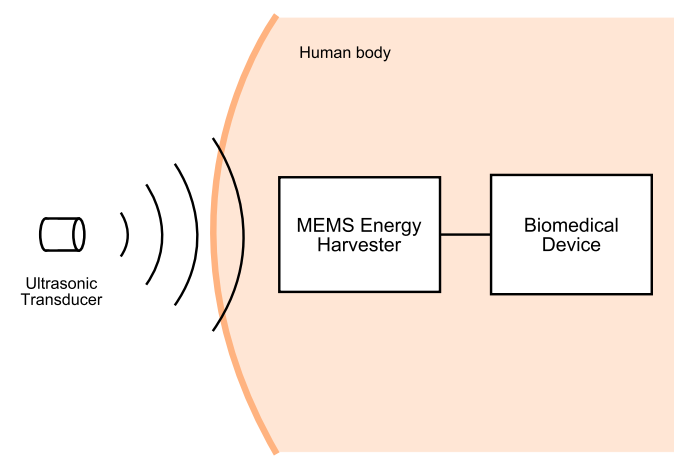

Fig. 1. Overview of MEMS ultrasonic energy harvesting.

to these devices [4]. The integration of a MEMS energy harvester with such biomedical devices is a potential solution to this issue. As shown in [5], ultrasonic waves can effectively be used to mechanically excite an implanted MEMS device, meaning that a microscale energy harvester could be used to power an implanted system through harvesting of the resulting mechanical vibrations (Fig. 1).

This letter presents a MEMS energy harvester that uses ultrasonic waves for mechanical excitation with the harvested power being used to charge a load capacitor. Triangular electrostatic electrodes are used to perform the energy conversion process, which are shown to be more effective than conventional electrostatic comb fingers.

\section{Energy Harvester Design And Fabrication}

\section{A. 2-DOF Ultrasonic Energy Harvester Design}

The MEMS energy harvester is largely derived from the system described in [6]-[8]. The device features a central proof mass approximately $1 \times 1 \mathrm{~mm}$ in size, which is mechanically excited by incident ultrasonic waves. The device features two mechanical degrees of freedom (2-DOF) and uses a parallel kinematic structure with beam flexures connected to the corners of the mass allowing it to vibrate in the in-plane $x$ and $y$ directions. These flexures decouple the $x$ and $y$ components of the vibrations of the proof mass with the displacements being harvested via separate variable capacitors located at the edges of the device.

To maximize the vibrations of the proof mass during operation, the harvester is designed such that its first mechanical 


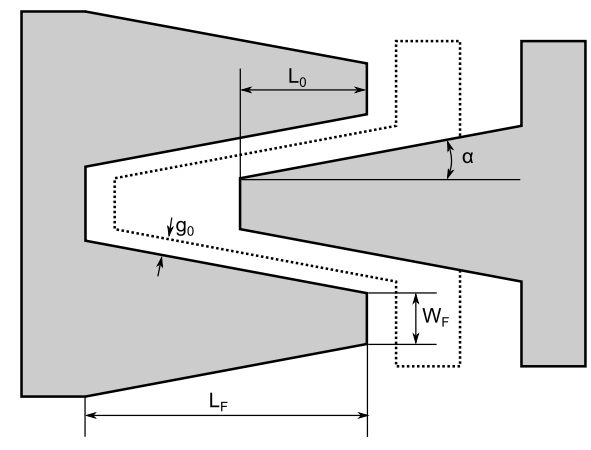

Fig. 2. Triangular electrostatic electrode.

resonance along the in-plane directions is closely matched with the frequency of the ultrasonic waves produced by the transmitter used to excite the device. These resonance modes are located at $\sim 40 \mathrm{kHz}$, which is a common frequency for commercially available ultrasonic transmitters.

\section{B. Triangular Electrostatic Electrodes}

To convert the vibrations of the proof mass into capacitance variations to facilitate the harvesting of electrical energy, the MEMS energy harvester presented in [7] features conventional overlap-varying electrodes. These are in the form of parallel comb fingers with a finger width and interfinger gap of $3 \mu \mathrm{m}$. However, the nonuniform shaping of comb fingers can significantly influence the properties of electrostatic electrodes in both nanopositioning and energy harvesting applications [9]-[11]. In particular, an appropriately designed triangular electrode is able to provide both a larger total capacitance change compared with conventional gap-closing electrodes and a greater change in capacitance per unit displacement compared with overlap-varying electrodes. A triangular electrode structure may therefore result in a more effective transduction mechanism for electrostatic energy harvesting devices.

The structure of a triangular electrode may be defined by a number of parameters, including the initial finger overlap $L_{0}$, the initial gap between fingers $g_{0}$, the finger length $L_{F}$, the width at the end of the finger $W_{F}$, and the finger sidewall angle $\alpha$ [11] (see Fig. 2). Although some of these parameters such as $g_{0}$ are dependent on the minimum feature size of the fabrication process, other parameters, including $L_{0}$ and $\alpha$ are optimally chosen based on the maximum displacement of the harvester's mechanical structure. To maximize the total change in capacitance of the MEMS energy harvester's triangular electrodes, the experimentally obtained characterization data for the earlier device presented in [7] was utilized. The electrode parameters were chosen based on the harvester displacement previously observed, and they are listed in Table I.

\section{Fabrication}

The energy harvester was fabricated using the commercial silicon-on-insulator SOIMUMPs fabrication process provided by MEMSCAP [12]. This is a SoI process with the main features of the harvester, including the proof mass, flexures, and triangular electrodes being comprised of $25-\mu \mathrm{m}$-thick
TABLE I

Triangular Electrode Design Parameters

\begin{tabular}{|c|c|}
\hline Finger length $\left(L_{0}\right)$ & $5 \mu \mathrm{m}$ \\
\hline Initial finger gap $\left(g_{0}\right)$ & $3 \mu \mathrm{m}$ \\
\hline Finger length $\left(L_{F}\right)$ & $11 \mu \mathrm{m}$ \\
\hline Finger end width $\left(W_{F}\right)$ & $3 \mu \mathrm{m}$ \\
\hline Finger sidewall angle $(\alpha)$ & $23.6^{\circ}$ \\
\hline
\end{tabular}

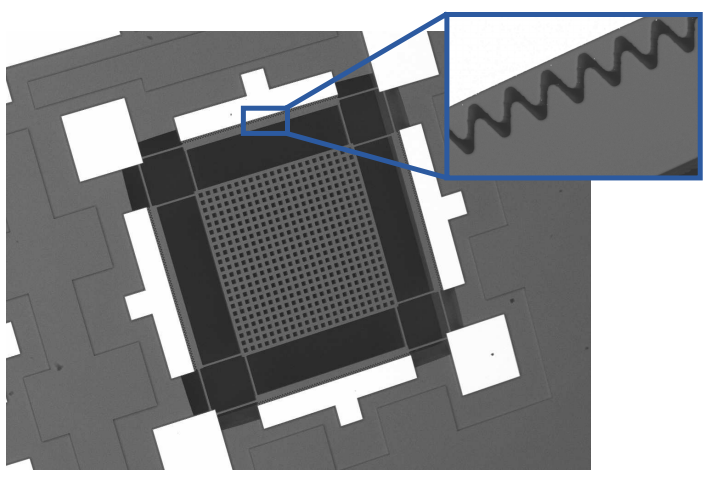

Fig. 3. SEM image of fabricated MEMS energy harvester.

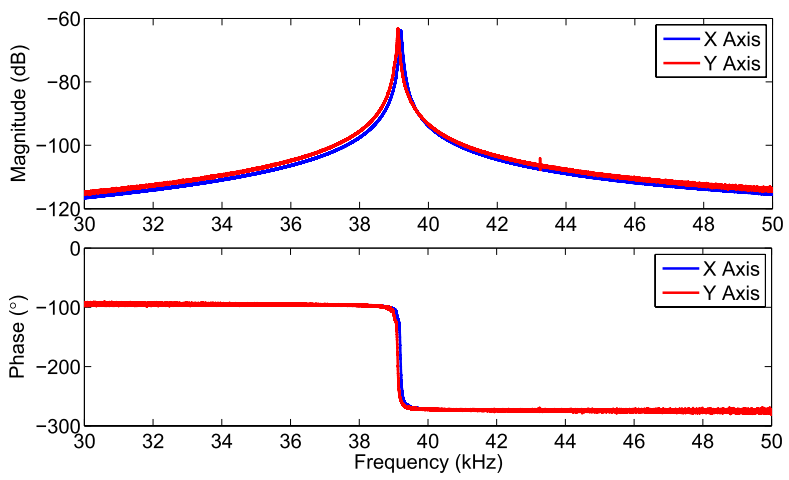

Fig. 4. Frequency response of mechanical system.

doped silicon. Scanning electron microscope (SEM) images of the fabricated energy harvester are shown in Fig. 3.

\section{EXPERIMENTAL CHARACTERIZATION}

To experimentally identify the mechanical resonance modes of the fabricated MEMS energy harvester, a Polytec MSA050-3D microsystem analyzer (MSA) was used to perform a frequency response analysis. The mechanical structure was excited by providing a $60 \mathrm{~V}$ dc bias to the harvester's electrodes together with a small amplitude chirp signal. In a practical implementation, the required electrical bias would be implemented through methods, such as the use of electret materials with a quasi-permanent electric charge, or using additional power conversion circuitry to provide the bias from the harvested electrical power. For testing and characterization purposes however, the use of an external bias is sufficient. As shown in Fig. 4, the first resonance modes of the harvester are at 39.20 and $39.12 \mathrm{kHz}$ for the $x$-axis and $y$-axis, respectively.

The electrical output of the harvester was analyzed using an SR780 signal analyzer, with an SQ40T ultrasonic transducer being used to excite the device. The transducer was placed 


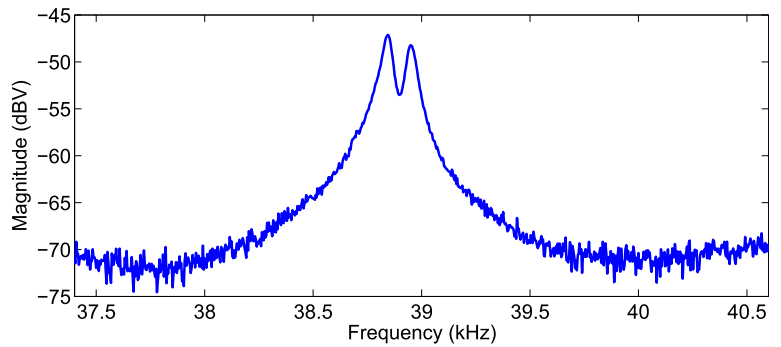

Fig. 5. Magnitude plot of energy harvester frequency response obtained using a signal analyzer.

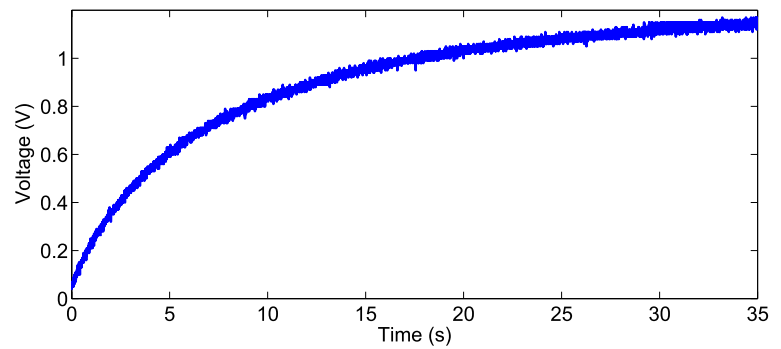

Fig. 6. Rising voltage on storage capacitor due to harvesting of ultrasonic waves.

approximately $5 \mathrm{~cm}$ away from the MEMS die, which is similar to the distance that would be involve in the powering of an implanted device. A second frequency response analysis was performed, this time using the electrical power generated by the harvester. The positive $x$ and $y$ electrodes of the MEMS harvester were biased with $100 \mathrm{~V}$ DC, while the ultrasonic transmitter was driven by a $2 \mathrm{~V}$ sinusoidal signal and positioned at a $45^{\circ}$ angle to the $x$ and $y$ axes of the harvester. The results of this frequency response analysis are displayed in Fig. 5. As shown there are two clear resonance peaks at 38.84 and $38.95 \mathrm{kHz}$, corresponding to the resonance modes of the $y$ and $x$ axes of the device. These frequencies are slightly lower than those measured using the MSA, which can be attributed to the application of a higher electrical bias in the second test. As noted in [11], the triangular electrode structure tends to produce a spring softening effect that is dependent on the applied electrical bias, therefore slightly reducing the inplane natural frequencies of the system during operation.

The power output of the energy harvester was assessed by connecting the device to a diode-based rectifying circuit and a $1-\mu \mathrm{F}$ storage capacitor. The electrostatic transducers were biased with $60 \mathrm{~V}$ dc, whereas the ultrasonic transmitter was driven by a $5 \mathrm{~V}$ sinusoidal signal at the $38.95-\mathrm{kHz}$ resonance frequency of the harvester's $x$-axis. The voltage on the storage capacitor was measured by an oscilloscope connected via a Stanford SR560 low-noise voltage preamplifier. When the transmitter is activated, the mechanical excitation of the MEMS energy harvester results in the capacitor being charged with the change in voltage being shown in Fig. 6. Over the initial 15-s period, the voltage increases from 0.04 to $0.95 \mathrm{~V}$, which corresponds to an average power output of $27.6 \mathrm{nW}$. With all other experimental testing variables being equal, this represents a $29 \%$ improvement over the power figure shown in [7].

\section{CONCLUSION}

In this letter, a novel 2-DOF MEMS energy harvester for biomedical applications has been presented. The device uses an external source of ultrasonic waves to mechanically excite a proof mass with electrostatic transducers being used to convert these vibrations into electrical power. The device is based upon a previously reported design [7] but features optimized triangular electrostatic electrode structures, which have been shown to provide greater capacitance variations than conventional parallel finger electrodes [11].

Experimental characterization of the fabricated harvester shows that the device is able to harvest $27.6 \mathrm{nW}$ of electrical power when excited by a source of ultrasonic waves at its mechanical resonance frequency of $38.95 \mathrm{kHz}$. This represents a $29 \%$ increase in harvested power over the existing design, increasing the potential effectiveness of the device as part of an implanted biomedical system.

As a proof-of-concept system, the device highlights the potential effectiveness of MEMS-based harvesters for implanted devices. Although challenges remain in developing a practical implementation of the device, including efficiently coupling ultrasonic energy from human tissue into the harvester, there is also significant scope for increasing the power output of the system. These considerations are likely to be addressed in future research.

\section{REFERENCES}

[1] S. Roundy, P. K. Wright, and J. Rabaey, "A study of low level vibrations as a power source for wireless sensor nodes," Comput. Commun., vol. 26, no. 11, pp. 1131-1144, Jul. 2003.

[2] P. D. Mitcheson, E. M. Yeatman, G. K. Rao, et al., "Energy harvesting from human and machine motion for wireless electronic devices," Proc. IEEE, vol. 96, no. 9, pp. 1457-1486, Sep. 2008.

[3] S. P. Beeby, M. J. Tudor, and N. M. White, "Energy harvesting vibration sources for microsystems applications," Meas. Sci. Technol., vol. 17, no. 12, pp. 175-195, 2006.

[4] S. Arra, J. Leskinen, J. Heikkila, et al., "Ultrasonic power and data link for wireless implantable applications," in Proc. 2nd Int. Symp. Wireless Pervas. Comput., Feb. 2007.

[5] A. Denisov and E. Yeatman, "Stepwise microactuators powered by ultrasonic transfer," Procedia Eng., vol. 25, pp. 685-688, 2011.

[6] Y. Zhu, S. O. R. Moheimani, and M. R. Yuce, "A 2-DOF wideband electrostatic transducer for energy harvesting and implantable applications," in Proc. IEEE Sensors Conf., Christchurch, New Zealand, Oct. 2009, pp. 1542-1545.

[7] Y. Zhu, S. O. R. Moheimani, and M. R. Yuce, "Ultrasonic energy transmission and conversion using a 2-D MEMS resonator," IEEE Electron Device Lett., vol. 31, no. 4, pp. 374-376, Apr. 2010.

[8] Y. Zhu, S. O. R. Moheimani, and M. R. Yuce, "A 2-DOF MEMS ultrasonic energy harvester," IEEE Sensors J., vol. 11, no. 1, pp. 155-161, Jan. 2011.

[9] B. D. Jensen, S. Mutlu, S. Miller, et al., "Shaped comb fingers for tailored electromechanical restoring force," J. Microelectromech. Syst., vol. 12, no. 3, pp. 373-383, 2003.

[10] J. B. C. Engelen, M. A. Lantz, H. E. Rothuizen, et al., "Improved performance of large stroke comb-drive actuators by using a stepped finger shape," in Proc. Int. Solid-State Sensors, Actuat. Microsyst. Conf., 2009, pp. 1762-1765.

[11] D. Hoffmann, B. Folkmer, and Y. Manoli, "Analysis and characterization of triangular electrode structures for electrostatic energy harvesting," J. Micromech. Microeng., vol. 21, no. 10, pp. 104002-1-104002-10, Oct. 2011.

[12] A. Cowen, G. Hames, D. Monk, S. Wilcenski, et al.. (2011). SOIMUMPs Design Handbook (Revision 8.0) [Online]. Available: http://www.memscap.com 